\title{
Study on Socio- Economic Profile of Farmers in Prayagraj District of Eastern Uttar Pradesh, India
}

\author{
Vikram Singh $^{1^{*}}$ and Ramchandra ${ }^{2}$
}

${ }^{1}$ College of Forestry, SHUATS, Prayagraj U. P.-211007, India

${ }^{2}$ Naini Agricultural Institute, SHUATS, Prayagraj, U. P., India

*Corresponding author

Keywords

Non agroforestry, Farmers, Villages,

Cultivating,

Agriculture, Issues, Planet

Article Info

Accepted:

12 October 2019

Available Online:

10 November 2019
The study was conducted in eight villages of Prayagraj adopting non agroforestry based farming system of Uttar Pradesh. The sample size 160 farmers of two block and 8 villages cultivating agriculture crop in Prayagraj district. The findings of the study revealed that, the non agroforestry farmers $43.49 \%$ had middle age (30-50 yrs.), $80.84 \%$ had literate, $44.68 \%$ were medium farming experience (11 to 20 years), $48.20 \%$ were medium family size (5-7 members), $68.45 \%$ were nuclear family type, $46.36 \%$ were semi cemented house type, $44.38 \%$ were medium farmer (1 to 2 hectare) land holding, $38.44 \%$ were medium income group per year (Rs.26000 to 50000), mass media participation are less than 50\% respondents. As far as extension participation is concerned about non agroforestry farmers very less participation in different types of programmes. The average risk orientation of non agroforestry respondents showed that majority of the $50.96 \%$ were low risk orientation, $57.03 \%$ were medium scientific orientation.

\section{Introduction}

One of the most widespread anthropogenic changes affecting the planet is forest conversion for alternative human use, resulting in environmental degradation and climate change. Farmers depending on subsistence agriculture are most vulnerable to the effects of environmental degradation and climate change, since their lack of economic resources restricts access to alternative livelihoods (Sling et al., 2005). As a result, per capita food output has declined in sub-Saharan Africa and the region has the highest proportion of undernourished people in the world, estimated to be $30 \%$ of the total population or 239 million people in 2010 (FAO 2010). There is a serious need for 
sustainable agricultural practices that can address these issues. In recent decades, there has been an increased focus on sustainable intensification in African agriculture. (Pretty et al., 2011)

An important objective of rural development is to increase agricultural productivity with a view to enhancing farmer's income and standard of living. Improved practices provide the main venue for increasing productivity in the country's agriculture (Edna et al., 2009).

Human resource is the most precious resource for any country. It is, however, not the numerical but the qualitative strength of the people which forges a country ahead towards progress and prosperity. It is basically the development of human resources that brings about socio-economic or political-cultural transformation of any society (Dubey and Srivastava, 2007).

Though agriculture was the principal sector in the state, its output expansion has been low and dormant for the past three decades. During the $1980 \mathrm{~s}$, agriculture recorded a 2.43 per cent growth rate, which slightly reduced to 2.40 per cent in the first decade of the 21st century (Tripathi and Mishra 2017). Agriculture output growth had at all times been lower than the all-India average since the 1980s in UP (Tripathi and Mishra 2017) even though it had strong agriculture support being the biggest producer of food grains, pulses, sugarcane and potato (Tripathi and Mishra 2017).

The state ranks first in both wheat and sugarcane production, third in both rice and pulse production and it also ranks first in potato production. Despite this, the output per hectare of nearly all crops in the state is average (GoI, 2011); the output per hectare for not a sole crop was the highest in the country. The productivity of every crop depends on the size of land holdings, farm mechanization and irrigation, utilization of fertilizer and use of high yield variety seeds. Excepting its western region, farming practices in the rest of the state are deficient in the above-mentioned productivity factors, which may be the major reason for the average yield per hectare for approximately all crops in the state.

\section{Materials and Methods}

The present study was designed to know the socio-economic characteristics of non agroforestry growers regarding adoption of improved production technology, with the following specific objective. To study the socio- economic profile of sampled non agroforestry growers in study area. Based on preliminary studies, the districts in the Uttar Pradesh were selected regions where farmers adopted non agroforestry practices. The present study was conducted in villages are Bagbana, Champatpur, Bigahiya, Bhandra and Baroli, Panwari, Bari bajahia, Panvar village of Chaka and Kaundhiyara block respectively, in Prayagraj district of Uttar Pradesh generally adopting non agroforestry system during 2017 to 2018 .

Adopting multi-stage random sampling one district, two blocks and four villages per block were selected from Prayagraj non agroforestry regions. In each of the selected villages, 20 farmers with some form of non agroforestry practices were selected. From the non agroforestry cultivation Prayagraj district was selected purposely. Thus a total of two blocks and 8 villages and 160 farmers formed the sample units for the study. The farmers' data was post stratified to compare across the farmholding size classes. The data were collected with the help of personal interview questionnaire survey method during the study period 2017 and 2018. The data were analyzed, tabulated and the results were drawn with the help of appropriate statistical methods. 


\section{Results and Discussion}

The results of the study are presented socioeconomic conditions of the adopting of the non agroforestry cultivation in the study area during 2017-18. Prayagraj district farmers are having proper knowledge and adoption about scientific and technical knowledge of cultivation practices.

\section{Land holding}

Study depicts that majority $44.38 \%$ were found in the land holding category of medium farmers ( 1 to $2 \mathrm{ha}$ ) followed by $32.50 \%$ and $23.13 \%$ in the categories of small farmers (up to 1 ha) and large farmers (> 2 ha) respectively which are adopted non agroforestry system in Prayagraj district. This is due to the fact that in Prayagraj per capita agricultural land is comparatively less. Singh et al., (2009)

\section{Age}

The table 1 showed that the maximum 46.15 and 46.48 per cent of respondents belonged to 30 to 50 years age group of small and medium farmers respectively and 45.95 percent of respondents belonged to above 50 years age group of large farmers which are adopted non agroforestry system in Prayagraj district.

\section{Education}

The table 1 showed that the maximum 86.54 , 80.28 and 75.68 per cent of respondents were literate of small, medium and large farmers respectively which are adopted non agroforestry system in Prayagraj district. Chakraborty et al., (2015)

\section{Farming experience}

The table 1 showed that the maximum 44.23, 49.29 and 40.54 per cent were medium farming experience (11 to $20 \mathrm{yrs}$.) of small, medium and large farmers respectively which are adopted non agroforestry system in Prayagraj district. Dwivedi et al., (2007)

\section{Family size}

The table 1 showed that the maximum 48.08 , 47.88 and 48.65 per cent were medium family size (5 to 7 members) of small, medium and large farmers respectively which are adopted non agroforestry system in Prayagraj district. Singh et al., (2017)

\section{Type of family}

The table 1 showed that the maximum 36.54 , 30.98 and 27.03 per cent were belong to joint family of the total sample of small, medium and large farmers respectively which are adopted non agroforestry system in Prayagraj district. Chauhan et al., (2017)

\section{House type}

The table 1 showed that the maximum 48.08 , 45.07 and 45.95 per cent were semi cemented house type of the total sample of small, medium and large farmers respectively which are adopted non agroforestry system in Prayagraj district. Chakraborty et al., (2015)

\section{Annual income}

The table 1 reveals that the annual income of $84.62 \%$ respondents majority was found in the low category of (up to Rs. 25000) in small farmers group, $76.06 \%$ respondents majority was found in the medium category of (Rs. 26000 - Rs. 50000) in medium farmers group and $54.05 \%$ respondents majority was found in the high category of (above Rs. 50000) in large farmers group which are adopted non agroforestry system in Prayagraj district. Maurya et al., (2018) 


\section{Inventory status}

The table 1 reveals that the material posses by small farmers $63.46 \%, 63.46 \%$ and $26.92 \%$ respondents have radio/TV/mobile, cycle and scooter/motor bike respectively, by medium farmers $85.92 \%, 60.56 \%, 47.89 \%, 05.63 \%$ and $05.63 \%$ have radio/TV/mobile, cycle, scooter/motor bike, car/tractor and improved agriculture machineries respectively and by large farmers $100.00 \%, 35.14 \%, 64.86 \%$, $21.62 \%$ and $21.62 \%$ have $\mathrm{radio} / \mathrm{TV} / \mathrm{mobile}$, cycle, scooter/motor bike, car/tractor and improved agriculture machineries respectively which are adopted non agroforestry system in Prayagraj district. Singh et al., (2009)

\section{Risk orientation}

The table 1 showed that maximum $38.03 \%$ and $43.24 \%$ of respondents belonged to low level of risk bearing ability of medium and large farmers respectively and 53.85 per cent of respondents belonged to medium level of risk bearing ability of small farmers which are adopted non agroforestry system in Prayagraj district. Tinde et al., (2017)

\section{Scientific orientation}

The table 1 showed that maximum $69.23 \%$, $50.70 \%$ and $51.35 \%$ of respondents belonged to medium level of scientific orientation of small, medium and large farmers respectively which are adopted non agroforestry system in Prayagraj district. Tinde et al., (2017)

\section{Mass media participation}

The data presented of Prayagraj respondents in table 3 revealed that by small farmers most of the respondents viewed television 7.69 percent of respondents regularly, 28.85 percent respondents occasionally and 63.46 percent never viewed agriculture programmes. By the medium farmers 09.86 percent of respondents regularly, 30.99 percent respondents occasionally and 59.15 percent never viewed agriculture programmes. By the large farmers 13.51 percent of respondents regularly, 27.03 percent respondents occasionally and 59.46 percent never viewed agriculture programmes.

So majority of the sampled farmers used it for watching the general programme than the agriculture programmes

\section{Extension participation}

The data presented of Prayagraj respondents in table 2 revealed that by small farmers most of the respondents attended the group meetings (90.38\%), followed by the demonstrations $(63.46 \%)$, field visits $(51.92 \%)$, field days $(46.16 \%)$, training programmes $(42.31 \%)$, agriculture exhibitions (44.23\%), krishi melas $(38.46 \%)$ and educational tours $(34.62 \%)$ respectively. By medium farmer's most of the respondents attended the group meetings (87.32\%), followed by the demonstrations $(67.61 \%)$, field visits $(54.93 \%)$, training programmes (54.93\%), krishi melas (53.52\%), agriculture exhibitions (53.52\%), field days $(46.48 \%)$, and educational tours $(36.62 \%)$ respectively. By large farmers' group meetings $(89.19 \%)$, followed by the krishi melas (67.57\%), demonstrations (64.86\%), field visits $(64.86 \%)$, agriculture exhibitions $(54.05 \%)$, field days $(40.54 \%)$, training programmes $(40.54 \%)$ and educational tours $(35.14 \%)$ respectively which are adopted non agroforestry system. Safa et al., (2005)

The socio-economic characteristics of farmers are important for better policy options. The result of the study, indicated that non agroforestry farmers of Prayagraj district, Uttar Pradesh had not proper knowledge and adoption about scientific cultivation practices and as well as about agroforestry practices knowledge in comparison to other techniques adopting farmers. 
Table.1 Socioeconomic status of land holdings wise classification of respondents of Kaushambi districts

\begin{tabular}{|c|c|c|c|c|c|c|c|c|}
\hline \multirow[t]{3}{*}{ S. no } & \multirow[t]{3}{*}{ Variable } & \multirow[t]{3}{*}{ Category } & \multicolumn{6}{|c|}{ Prayagraj (Non agroforestry respondents) } \\
\hline & & & \multicolumn{2}{|c|}{ Small farmers } & \multicolumn{2}{|c|}{ Medium farmers } & \multicolumn{2}{|c|}{ Large farmers } \\
\hline & & & $\mathbf{F}$ & $\mathbf{P}$ & $\mathbf{F}$ & $\mathbf{P}$ & $\mathbf{F}$ & $\mathbf{P}$ \\
\hline \multirow[t]{3}{*}{1.} & \multirow[t]{3}{*}{ Land holdings } & up to 1 ha & 52.00 & 32.50 & - & - & - & - \\
\hline & & 1 to 2 ha & - & - & 71.00 & 44.38 & - & - \\
\hline & & More than 2 ha & - & - & - & - & 37.00 & 23.13 \\
\hline \multirow[t]{6}{*}{2.} & \multirow{6}{*}{$\begin{array}{l}\text { Age } \\
\text { (yrs) }\end{array}$} & Young $(<29)$ & 9.00 & 17.31 & 26.00 & 36.62 & 6.000 & 16.22 \\
\hline & & Middle (30-50) & 24.00 & 46.15 & 33.00 & 46.48 & 14.00 & 37.84 \\
\hline & & Old $(>50)$ & 19.00 & 36.54 & 12.00 & 16.90 & 17.00 & 45.95 \\
\hline & & Total & 52.00 & 100.00 & 71.00 & 100.00 & 37.00 & 100.00 \\
\hline & & Mean & \multicolumn{2}{|c|}{44.212} & \multicolumn{2}{|c|}{38.310} & \multicolumn{2}{|c|}{46.027} \\
\hline & & $\mathrm{SE} \pm \mathrm{d}$ & \multicolumn{2}{|c|}{11.212} & \multicolumn{2}{|c|}{10.611} & \multicolumn{2}{|c|}{10.795} \\
\hline \multirow[t]{9}{*}{3.} & \multirow[t]{9}{*}{ Education } & Illiterate & 07.00 & 13.46 & 14.00 & 19.72 & 09.00 & 24.32 \\
\hline & & Primary school & 13.00 & 25.00 & 14.00 & 19.72 & 05.00 & 13.51 \\
\hline & & Middle school & 16.00 & 30.77 & 19.00 & 26.76 & 11.00 & 29.73 \\
\hline & & High school & 08.00 & 15.38 & 12.00 & 16.90 & 08.00 & 21.62 \\
\hline & & Intermediate & 06.00 & 11.54 & 10.00 & 14.08 & 03.00 & 08.11 \\
\hline & & Above intermediate & 02.00 & 03.85 & 02.00 & 02.82 & 01.00 & 02.70 \\
\hline & & Total & 52.00 & 100.00 & 71.00 & 100.00 & 37.00 & 100.00 \\
\hline & & Mean & \multicolumn{2}{|c|}{1.981} & \multicolumn{2}{|c|}{1.944} & \multicolumn{2}{|c|}{1.838} \\
\hline & & SE. $\pm d$. & \multicolumn{2}{|c|}{1.336} & \multicolumn{2}{|c|}{1.413} & \multicolumn{2}{|c|}{1.385} \\
\hline \multirow[t]{6}{*}{4.} & \multirow{6}{*}{$\begin{array}{l}\text { Agriculture/agrof } \\
\text { orestry farming } \\
\text { experience }\end{array}$} & Low (up to10 years) & 10.00 & 19.23 & 19.00 & 26.76 & 09.00 & 24.32 \\
\hline & & Medium (11 to 20 years) & 23.00 & 44.23 & 35.00 & 49.29 & 15.00 & 40.54 \\
\hline & & High ( > 21 years ) & 19.00 & 36.54 & 17.00 & 23.94 & 13.00 & 35.14 \\
\hline & & Total & 52.00 & 100.00 & 71.00 & 100.00 & 37.00 & 100.00 \\
\hline & & Mean & \multicolumn{2}{|c|}{17.231} & \multicolumn{2}{|c|}{14.366} & \multicolumn{2}{|c|}{16.459} \\
\hline & & SE. $\pm d$. & \multicolumn{2}{|c|}{06.850} & & & & \\
\hline
\end{tabular}




\begin{tabular}{|c|c|c|c|c|c|c|c|c|}
\hline \multirow[t]{6}{*}{5.} & \multirow[t]{6}{*}{ Family size } & Small (up to 4 members) & 20.00 & 38.46 & 25.00 & 35.21 & 14.00 & 37.84 \\
\hline & & Medium (5-7 members) & 25.00 & 48.08 & 34.00 & 47.88 & 18.00 & 48.65 \\
\hline & & Large (>7 members) & 07.00 & 13.46 & 12.00 & 16.90 & 05.00 & 13.51 \\
\hline & & Total & 52.00 & 100.00 & 71.00 & 100.0 & 37.00 & 100.00 \\
\hline & & Mean & \multicolumn{2}{|c|}{05.346} & \multicolumn{2}{|c|}{05.479} & \multicolumn{2}{|c|}{05.459} \\
\hline & & SE. \pm d. & \multicolumn{2}{|c|}{01.558} & \multicolumn{2}{|c|}{01.672} & \multicolumn{2}{|c|}{01.643} \\
\hline \multirow[t]{5}{*}{6.} & \multirow[t]{5}{*}{ Family type } & Nuclear & 33.00 & 63.46 & 49.00 & 69.02 & 27.00 & 72.97 \\
\hline & & Joint & 19.00 & 36.54 & 22.00 & 30.98 & 10.00 & 27.03 \\
\hline & & Total & 52.00 & 100.00 & 71.00 & 100.0 & 37.00 & 100.00 \\
\hline & & Mean & \multicolumn{2}{|c|}{01.154} & \multicolumn{2}{|c|}{01.155} & \multicolumn{2}{|c|}{01.243} \\
\hline & & SE. $\pm d$. & \multicolumn{2}{|c|}{0.364} & \multicolumn{2}{|c|}{0.364} & \multicolumn{2}{|c|}{0.435} \\
\hline \multirow[t]{6}{*}{7.} & \multirow[t]{6}{*}{ House type } & Kutchcha & 19.00 & 36.54 & 27.00 & 38.03 & 14.00 & 37.84 \\
\hline & & Semi cemented & 25.00 & 48.08 & 32.00 & 45.07 & 17.00 & 45.95 \\
\hline & & Cemented & 08.00 & 15.38 & 12.00 & 16.90 & 06.00 & 16.22 \\
\hline & & Total & 52.00 & 100.00 & 71.00 & 100.00 & 37.00 & 100.00 \\
\hline & & Mean & \multicolumn{2}{|c|}{01.788} & \multicolumn{2}{|c|}{01.789} & \multicolumn{2}{|c|}{01.703} \\
\hline & & SE. $\pm d$. & \multicolumn{2}{|c|}{0.696} & \multicolumn{2}{|c|}{0.715} & \multicolumn{2}{|c|}{0.740} \\
\hline \multirow[t]{6}{*}{8.} & \multirow[t]{6}{*}{ Annual income } & Low (up to $\quad$ Rs. 25000) & 44.00 & 84.62 & 14.00 & 19.72 & 00.00 & 00.00 \\
\hline & & Medium (Rs. 26000-50000) & 08.00 & 15.38 & 54.00 & 76.06 & 17.00 & 45.95 \\
\hline & & High (Above Rs. 50000) & 00.00 & 00.00 & 03.00 & 04.23 & 20.00 & 54.05 \\
\hline & & Total & 52.00 & 100.0 & 71.00 & 100.00 & 37.00 & 100.00 \\
\hline & & Mean & \multicolumn{2}{|c|}{22346.15} & \multicolumn{2}{|c|}{29985.91} & \multicolumn{2}{|c|}{47432.4} \\
\hline & & SE. $\pm d$. & \multicolumn{2}{|c|}{4096.314} & \multicolumn{2}{|c|}{7168.369} & & 66 \\
\hline & Inventory status & Radio/TV/Mobile & 33.00 & 63.46 & 61.00 & 85.92 & 37.00 & 100.00 \\
\hline 9. & & Cycle & 33.00 & 63.46 & 43.00 & 60.56 & 13.00 & 35.14 \\
\hline & & Scooter/Motor bike & 14.00 & 26.92 & 34.00 & 47.89 & 24.00 & 64.86 \\
\hline & & Car/tractor & 00.00 & 00.00 & 04.00 & 05.63 & 08.00 & 21.62 \\
\hline & & Improved agriculture & 00.00 & 00.00 & 04.00 & 05.63 & 08.00 & 21.62 \\
\hline
\end{tabular}




\begin{tabular}{|c|c|c|c|c|c|c|c|c|}
\hline & & machineries & & & & & & \\
\hline & & Mean & \multicolumn{2}{|c|}{16.00} & \multicolumn{2}{|c|}{29.20} & \multicolumn{2}{|c|}{18.00} \\
\hline & & SE. $\pm d$. & \multicolumn{2}{|c|}{16.54} & \multicolumn{2}{|c|}{24.97} & \multicolumn{2}{|c|}{12.47} \\
\hline \multirow[t]{6}{*}{10} & \multirow[t]{6}{*}{ Risk orientation } & Low & 15.00 & 28.85 & 27.00 & 38.03 & 16.00 & 43.24 \\
\hline & & Medium & 28.00 & 53.85 & 20.00 & 28.17 & 11.00 & 29.73 \\
\hline & & High & 09.00 & 17.30 & 24.00 & 33.80 & 10.00 & 27.03 \\
\hline & & Total & 52.00 & 100.00 & 71.00 & 100.00 & 37.00 & 100.00 \\
\hline & & Mean & \multicolumn{2}{|c|}{12.85} & \multicolumn{2}{|c|}{12.73} & \multicolumn{2}{|c|}{12.43} \\
\hline & & SE. $\pm d$. & \multicolumn{2}{|c|}{02.48} & \multicolumn{2}{|c|}{04.06} & \multicolumn{2}{|c|}{ 03.31 } \\
\hline \multirow[t]{6}{*}{11} & \multirow{6}{*}{$\begin{array}{c}\text { Scientific } \\
\text { orientation }\end{array}$} & Low & 13.00 & 25.00 & 17.00 & 23.94 & 03.00 & 08.11 \\
\hline & & Medium & 36.00 & 69.23 & 36.00 & 50.70 & 19.00 & 51.35 \\
\hline & & High & 03.00 & 05.77 & 18.00 & 25.35 & 15.00 & 40.54 \\
\hline & & Total & 52.00 & 100.00 & 71.00 & 100.0 & 37.00 & 100.00 \\
\hline & & Mean & \multicolumn{2}{|c|}{09.69} & \multicolumn{2}{|c|}{11.46} & \multicolumn{2}{|c|}{12.11} \\
\hline & & SE. $\pm d$. & \multicolumn{2}{|c|}{01.84} & \multicolumn{2}{|c|}{03.68} & \multicolumn{2}{|c|}{02.59} \\
\hline
\end{tabular}

Table.2 Mass media exposure of land holdings wise classification of respondents of Prayagraj districts

\begin{tabular}{|c|c|c|c|c|c|c|c|c|}
\hline \multirow[t]{3}{*}{ S. no. } & \multirow[t]{3}{*}{ Category } & \multirow[t]{3}{*}{ Mass media } & \multicolumn{6}{|c|}{ Prayagraj (Non agroforestry respondents) } \\
\hline & & & \multicolumn{2}{|c|}{ Regular } & \multicolumn{2}{|c|}{ Occasional } & \multicolumn{2}{|c|}{ Never } \\
\hline & & & $\mathbf{F}$ & $\mathbf{P}$ & $\mathbf{F}$ & $\mathbf{P}$ & $\mathbf{F}$ & $\mathbf{P}$ \\
\hline \multirow[t]{3}{*}{1} & \multirow[t]{3}{*}{ Small farmers } & Television & 04.00 & 07.69 & 15.00 & 28.85 & 33.00 & 63.46 \\
\hline & & Radio & 02.00 & 03.85 & 10.00 & 19.23 & 40.00 & 76.92 \\
\hline & & Newspaper & 04.00 & 07.69 & 12.00 & 23.08 & 36.00 & 69.23 \\
\hline \multirow[t]{3}{*}{2} & \multirow[t]{3}{*}{ Medium farmers } & Television & 07.00 & 09.86 & 22.00 & 30.99 & 42.00 & 59.15 \\
\hline & & Radio & 03.00 & 04.23 & 14.00 & 19.72 & 54.00 & 76.06 \\
\hline & & Newspaper & 09.00 & 12.68 & 15.00 & 21.13 & 47.00 & 66.20 \\
\hline \multirow[t]{3}{*}{3} & \multirow[t]{3}{*}{ Large farmers } & Television & 05.00 & 13.51 & 10.00 & 27.03 & 22.00 & 59.46 \\
\hline & & Radio & 01.00 & 02.70 & 07.00 & 18.92 & 29.00 & 78.38 \\
\hline & & Newspaper & 05.00 & 13.51 & 06.00 & 16.22 & 26.00 & 70.27 \\
\hline
\end{tabular}


Table.3 Extension participation of land holdings wise classification of respondents of Prayagraj districts

\begin{tabular}{|c|c|c|c|c|c|c|c|c|c|}
\hline \multirow{3}{*}{$\begin{array}{c}\text { S. } \\
\text { no. }\end{array}$} & \multirow[t]{3}{*}{ Category } & \multicolumn{8}{|c|}{ Prayagraj (Non agroforestry respondents) } \\
\hline & & \multicolumn{2}{|c|}{ Attended } & \multicolumn{2}{|c|}{ Regular } & \multicolumn{2}{|c|}{ Occasional } & \multicolumn{2}{|c|}{ Never } \\
\hline & & $\mathrm{F}$ & $\mathrm{P}$ & $\mathrm{F}$ & $\mathrm{P}$ & $\mathrm{F}$ & $\mathrm{P}$ & $\mathrm{F}$ & $\mathrm{P}$ \\
\hline A. & \multicolumn{9}{|c|}{ Small farmers } \\
\hline 1. & Training program & 22.0 & 42.31 & 03.0 & 05.77 & 19.0 & 36.54 & 30.0 & 57.69 \\
\hline 2. & Demonstration & 33.0 & 63.46 & 08.0 & 15.38 & 25.0 & 48.08 & 19.0 & 36.54 \\
\hline 3. & Field days & 24.0 & 46.16 & 02.0 & 03.85 & 22.0 & 42.31 & 28.0 & 53.85 \\
\hline 4. & Field visit & 27.0 & 51.92 & 07.0 & 13.46 & 20.0 & 38.46 & 25.0 & 48.08 \\
\hline 5. & Groups meeting & 47.0 & 90.38 & 20.0 & 38.46 & 27.0 & 51.92 & 05.0 & 09.62 \\
\hline 6. & Agri. exhibition & 23.0 & 44.23 & 08.0 & 15.38 & 15.0 & 28.85 & 29.0 & 55.77 \\
\hline 7. & Krishi mela & 20.0 & 38.46 & 07.0 & 13.46 & 13.0 & 25.00 & 32.0 & 61.54 \\
\hline 8. & Education tour & 18.0 & 34.62 & 02.0 & 03.85 & 16.0 & 30.77 & 34.0 & 65.38 \\
\hline & Mean & \multicolumn{2}{|c|}{26.75} & \multicolumn{2}{|c|}{07.10} & \multicolumn{2}{|c|}{19.60} & \multicolumn{2}{|c|}{25.30} \\
\hline & SE. $\pm d$. & \multicolumn{2}{|c|}{09.38} & \multicolumn{2}{|c|}{05.80} & \multicolumn{2}{|c|}{04.90} & \multicolumn{2}{|c|}{09.40} \\
\hline B. & \multicolumn{9}{|c|}{ Medium farmers } \\
\hline 1. & Training program & 39.0 & 54.93 & 06.0 & 08.45 & 33.0 & 46.48 & 32.0 & 45.07 \\
\hline 2. & Demonstration & 48.0 & 67.61 & 13.0 & 18.31 & 35.0 & 49.30 & 23.0 & 32.39 \\
\hline 3. & Field days & 33.0 & 46.48 & 04.0 & 05.63 & 29.0 & 40.85 & 38.0 & 53.52 \\
\hline 4. & Field visit & 39.0 & 54.93 & 15.0 & 21.13 & 24.0 & 33.80 & 32.0 & 45.07 \\
\hline 5. & Groups meeting & 62.0 & 87.32 & 28.0 & 39.44 & 34.0 & 47.89 & 09.0 & 12.68 \\
\hline 6. & Agri. exhibition & 38.0 & 53.52 & 14.0 & 19.72 & 24.0 & 33.80 & 33.0 & 46.48 \\
\hline 7. & Krishi mela & 38.0 & 53.52 & 14.0 & 19.72 & 24.0 & 33.80 & 33.0 & 46.48 \\
\hline 8. & Education tour & 26.0 & 36.62 & 07.0 & 09.86 & 19.0 & 26.76 & 45.0 & 63.38 \\
\hline & Mean & \multicolumn{2}{|c|}{40.37} & \multicolumn{2}{|c|}{12.60} & \multicolumn{2}{|c|}{27.80} & \multicolumn{2}{|c|}{30.60} \\
\hline & SE. $\pm d$. & \multicolumn{2}{|c|}{10.70} & & & \multicolumn{2}{|c|}{05.80} & \multicolumn{2}{|c|}{10.70} \\
\hline C. & \multicolumn{9}{|c|}{ Large farmers } \\
\hline 1. & Training program & 15.0 & 40.54 & 04.0 & 10.81 & 11.0 & 29.73 & 22.0 & 59.46 \\
\hline 2. & Demonstration & 24.0 & 64.86 & 06.0 & 16.22 & 18.0 & 48.65 & 13.0 & 35.14 \\
\hline 3. & Field days & 15.0 & 40.54 & 03.0 & 08.11 & 12.0 & 32.43 & 22.0 & 59.46 \\
\hline 4. & Field visit & 24.0 & 64.86 & 10.0 & 27.03 & 14.0 & 37.84 & 13.0 & 35.14 \\
\hline 5. & Groups meeting & 33.0 & 89.19 & 14.0 & 37.84 & 19.0 & 51.35 & 04.0 & 10.81 \\
\hline 6. & Agri. exhibition & 20.0 & 54.05 & 06.0 & 16.22 & 14.0 & 37.84 & 17.0 & 45.95 \\
\hline 7. & Krishi mela & 25.0 & 67.57 & 09.0 & 24.32 & 16.0 & 43.24 & 12.0 & 32.43 \\
\hline 8. & Education tour & 13.0 & 35.14 & 03.0 & 08.11 & 10.0 & 27.03 & 24.0 & 64.86 \\
\hline & Mean & \multicolumn{2}{|c|}{21.12} & & & & & & \\
\hline & SE. $\pm d$. & & & & & & & & \\
\hline
\end{tabular}


Economically less benefitted adopting non agroforestry cultivation of Prayagraj district farmers comparison to adopting agroforestry cultivation of other farmers. On the basis of the findings it is suggested that socioeconomic status of the Prayagraj district farmers which are not adopted agroforestry cultivation can be improved by adoption of agroforestry cultivation and imparting technical knowledge about agroforestry practices with increasing their education level and increasing their social participation and information sources.

\section{References}

Chakraborty, M., Haider, M. Z. and Rahaman, M. M., (2015). Socio-economic impact of cropland agroforestry: evidence from Jessore district of Bangladesh. Inter. J. Res. in Agric. and For., 2(1):11-20.

Chouhan, S., Daniel, S., David, A.A. and Paul, A. (2017). Analysis socioeconomic status of farmers adopted agroforestry of Basavanapura and Hejjige village, Nanjangud, India. Int. J. Curr. Microbiol. App. Sci. 6(7): 1745-1753.

Dwivedi, R.P., Tewari, R.K., Kareemulla, K., Chaturvedi, O.P. and Rai, P. (2007). Agri-horticultural system for household livelihood - a case study. Indian Res. J. Ext. Edu. 7(1).

Edna, C. Matthews-Njoku, Adesope, O.M. and Iruba, C. (2009). Acceptability of improved crop production practices among rural women in aguata agricultural zone of Anambra State, Nigeria. African J. Biotech. 8 (3): 405411.

Dubey, A. K. and Srivastava, J. P. (2007). Effect of training programme on knowledge and adoption behavior of farmers on wheat production technologies. Indian Res. Journal of Extension Education 7 (2\&3): 41-43.
FAO (2010). The state of food insecurity in the world. Rome: Food and Agriculture Organization of the United Nations.

Ganesh-Kumar, A., Mehta R., H. P., Sanjay, K., Prasad, K. G., Gulati, A. (2012). Demand and supply of cereals in India. IFPRI Discussion Paper 01158, New Delhi: International Food Policy Research Institute.

GoI (2011). "Agriculture Statistics at a Glance 2011." Directorate of Economics and Statistics, Ministry of agriculture, Government of India.

Greg, E. E., Bnam, B. E., William, M. F., \& Duru, E. J. C. (2011). Climate change, food security and agricultural productivity in African: Issues and policy directions. International Journal of Humanities and Social Science, 1, 205-223.

Kumar, S., Naresh, P. K., Aggarwal, D. N., Rani, R. S., Chauhan, N., \& Jain, S. (2014). Vulnerability of wheat production to climate change in India. Climate Research, 59, 173-187.

Maurya K., Kumar K., Kushwaha R. R. and Yadav R.S. (2018). An economic analysis of paddy cultivation and its processing in Mau district of eastern UP, India. Plant Archives. 18(1):11091112 .

Mishra, A. K., Tripathi, A. (2014). Wheat sector of India, paper presented at the workshop 'Eurasian wheat belt: future perspectives on regional and international food security', Istanbul, Turkey, 20-23 May 2014.

Pretty, J., Toulmin, C., and Williams, S., (2011). Sustainable intensification in African agriculture.

International journal of agricultural sustainability, 9 (1), 5-24.

Safa, M.S. (2005). Socio-economic factors affecting the income of small-scale agroforestry farms in hill country areas 
in Yemen: A comparison of OLS and WLS determinants. Small-scale Forest Economics, Management and Policy, 4(1): 117-134.

Singh D.K., Singh A.K., Yadav V.P., Singh R.B., Baghel R.S. and Singh M. (2009). Association of socio-economic status with economic motivation of the farmers. Indian Res. J Ext. Edu. 9(2), 2009.

Singh B.P., Yadav R.N., Mishra A.K., Gupta V., Mohammad M.R. and Singh N.K. (2017). A study of socio-economic status of guava orchardists in
Saharanpur district (Uttar Pradesh), India. Int. J. Curr. Microbiol. App. Sci. 6(8): 1845-1849.

Slingo, J.M., (2005). Introduction: food crops in a changing climate. Philosophical transactions of the royal society. Biological sciences, 360 (1463), 19831989.

Tripathi, A. and Mishra, A. K. (2017). Knowledge and passive adaptation to climate change: An example from Indian farmers. Climate Risk Management, Vol. 16: 195-207.

\section{How to cite this article:}

Vikram Singh and Ramchandra. 2019. Study on Socio- Economic Profile of Farmers in Prayagraj District of Eastern Uttar Pradesh, India. Int.J.Curr.Microbiol.App.Sci. 8(11): 14451454. doi: https://doi.org/10.20546/ijcmas.2019.811.170 